\title{
Decentralized Loading Coordinations for Large-population Plug-in Electric Vehicles and a few Controllable Bulk Loads
}

\author{
Xiaokun Yin \\ Zhongjing Ma \\ Lei Dong
}

\begin{abstract}
This paper studies the loading coordinations for large-population autonomous individual (plug-in) electric vehicles (EVs) and a few controllable bulk loads, e.g. EV fleets, pumped storage hydro units, and so on. Due to the computational infeasibility of the centralized coordination methods to the underlying large-population systems, in this paper we develop a novel game-based decentralized coordination strategy. Following the proposed decentralized strategy update mechanism and under some mild conditions, the system may quickly converge to a nearly valley-fill Nash equilibrium. The results are illustrated with numerical examples.
\end{abstract}

Key words: (Plug-in) electric vehicles, Loading coordination, Nash equilibrium (NE), Valley-fill.

\section{INTRODUCTION}

A number of recent studies have explored the potential impacts of high penetrations of (Plug-in) electric vehicles (EVs) on the power grid [1], [2], [3], [4]. In general, these studies assume that EV charging patterns "fill the valley" of nighttime demand. However, these studies do not address the issue of how to coordinate EV charging patterns. In [5], [6], [7], Ma, Duncan and Hiskens developed a strategy to coordinate the charging of large populations of autonomous EVs using concepts from non-cooperative games. The foundation of the work is a model that assumes EVs are cost-minimizing and weakly coupled via a common electricity price signal which is directly correlated with the grid network state, the number of EVs demanding resources and the urgency with which they demand it [8], and is different from grid system energy prices. At a Nash equilibrium, each EV reacts optimally with respect to a commonly observed charging trajectory that is the average of all EV strategies. This average is given by the solution of a fixed point problem in the limit of infinite population size. Accompanied by a decentralized computational algorithm the system converges to the nearly "valley-fill" Nash equilibrium in the infinite EV population limit.

This paper studies the loading coordinations for largepopulation autonomous individual EVs and a few controllable bulk loads, e.g. EV fleets, pumped storage hydros, and so on. Due to the computational infeasibility of the

Xiaokun Yin is with the School of Automation, Beijing Institute of Technology (BIT), Beijing, China, e-mail: yinxiaokun2005@163. com.

Zhongjing Ma (corresponding author) is with the School of Automation, BIT, and the Key Laboratory of Complex System Intelligent Control and Decision (BIT), Ministry of Education, Beijing, China, e-mail: mazhongjingebit. edu. cn.

Lei Dong is with the School of Automation, BIT, e-mail: motor. come163. com.

Research supported by the Excellent young scholars Research Fund of Beijing Institute of Technology. centralized coordination methods to the underlying largepopulation systems, in this paper we generalize the gamebased decentralized coordination strategy originally developed in [5], [6], [7]. We consider a model that all of the individual EV and bulk load agents, or called major agent, are cost-minimizing and coupled with each other via a common electricity price signal which is same as that applied in [5], [6], [7], and at any instance is determined by the total load in the grid at that instance.

As the number of the individual EVs grows substantially, the influence of each of the individual EVs on that aggregated load becomes negligible. Accordingly, in the infinite population limit, all EVs will observe the same average strategy of EV populations and the same total load as they calculate their local optimal charging strategy. Different from the individual EV demanding, in this paper we consider that the loading behavior of each of single major agents has significant influence on the grid with large populations of EVs.

In this situation, a collection of coordination strategies is a Nash equilibrium, if (i) each of the EVs reacts optimally with respect to a commonly observed charging trajectory that is the aggregation of all EV strategies and the strategies of all major agents [5]; and (ii) each of the major agents reacts optimally with respect to the EV aggregation strategy and the strategies of the rest of major agents. This aggregation strategy is an approximation of the solution to a fixed point problem in the limit of infinite EV populations.

Substantial work has been presented in the literature on the computation of Nash equilibria, or $\varepsilon$-Nash equilibria, for potential games which the loading coordination games studied in the paper belongs to, especially in relation to network games. Research on centralized mechanisms includes Christoudoulou et al. [9], who consider two classes of potential games, selfish routing games and cut games, and Even-Dar et al. [10] who study the number of steps required to reach a Nash equilibrium in load balancing games. Research on decentralized or distributed mechanisms includes Berenbrink [11], who propose a strongly distributed setting for load balancing games such that all agents update their strategy simultaneously. Also, Even-Dar et al. [12] present convergence results for an approximate $\varepsilon$-Nash equilibrium under a non-centralized setting in routing games, and Fischer et al. [13], [14] propose a distributed and concurrent process for convergence to Wardrop equilibria [15] in adaptive routing problems.

This paper presents a novel mixed computation procedure to implement the underlying NE. All of the individual EV 
agents simultaneously update their best individual strategy with respect to the strategies of all major agents and an average, or normalized aggregated, charging strategy of the whole EV population. This procedure continues until they do not update their best strategies any more. After the iterative procedure of EV populations, major agents successively updates his/her best strategy respectively with respect to the proposed aggregated strategy of EV populations, and loading behaviors of the rest of the major agent populations. The above procedure continues until neither individual EV and major agents update their best strategies any more. It is proposed that this procedure would be undertaken prior to the actual loading coordination interval. The computation time of this algorithm is unrelated to the number of EVs, since they simultaneously and independently update their charging strategy. Under certain mild conditions, the proposed best strategy update procedure drives the system asymptotically to a unique Nash equilibrium. In particular in the case of convex load supply curve and homogeneous EV populations, where all vehicles have identical parameters, a perfect valleyfill loading strategy is the Nash equilibrium. Several numerical examples are used to illustrate the performance of the solution strategy.

The paper is organized as follows. In Section II, we formulate loading coordination control problems for large populations of EVs and a few of major agents and design a mixed best strategy update mechanism and show that by applying this update mechanism and under some conditions, the system converges to a valley-fill Nash equilibrium. The results developed in Section II are explored with illustrative examples in Section III. In Section IV, we provide conclusions and suggest a few of possible extensions.

\section{LOADING COORDINATION PROBLEMS FOR LARGE-POPULATION EVS AND A FEW CONTROLLABLE BULK LOADS}

We consider the loading control problems of $N$ EVs and $M$ controllable bulk loads over a finite interval $\mathcal{T}$. We denote $\mathcal{M}$ and $\mathcal{N}$ as the set of bulk loads and the population of EVs respectively. We denote $u_{n t}^{E V}$ as the charging rate of individual EV $n \in \mathcal{N}$ at instant $t$, and $u_{m t}^{N, B L}$ as the charging rate of $m$ th bulk load unit, with $m \in \mathcal{M}$, at instant $t$ respectively. Moreover we call a charging strategy of individual EV $n$, with $n \in \mathcal{N}$, denoted $\mathbf{u}_{n}^{E V} \equiv\left(u_{n t}^{E V} ; t \in\right.$ $\mathcal{T})$, is admissible, if

$$
u_{n t}^{E V} \geq 0, \text { and }\left|\mathbf{u}_{n}^{E V}\right|_{1} \triangleq \sum_{t \in \mathcal{T}} u_{n t}^{E V}=\Gamma_{n}^{E V},
$$

where $\Gamma_{n}^{E V}$ denotes the maximum total loading capacity of EV $n$. Similarly we call a load strategy of individual bulk load $m$, with $m \in \mathcal{M}$, denoted $\mathbf{u}_{m}^{N, B L} \equiv\left(u_{m t}^{N, B L} ; t \in \mathcal{T}\right)$, is admissible, if

$$
u_{m t}^{N, B L} \geq 0, \text { and }\left|\mathbf{u}_{m}^{N, B L}\right|_{1} \triangleq \sum_{t \in \mathcal{T}} u_{m t}^{N, B L}=\Gamma_{m}^{N, B L},
$$

where $\Gamma_{m}^{N, B L}$ denotes the maximum total loading capacity of bulk load $m$.
We consider the following cost function subject to a collection of strategies $\mathbf{u} \equiv\left(\mathbf{u}_{n}^{E V}, \mathbf{u}_{m}^{N, B L} ; n \in \mathcal{N}, m \in \mathcal{M}\right)$ :

$$
J(\mathbf{u})=\sum_{t=1}^{T} p(t)\left(D_{t}^{N}+\sum_{n \in \mathcal{N}} u_{n t}^{E V}+\sum_{m \in \mathcal{M}} u_{m t}^{N, B L}\right)
$$

where $p(t)$ represents the electricity price at instant $t$, and $D_{t}^{N}$ denotes the inelastic base demand in the grid unrelated to the loading coordinations.

In this paper we suppose that $p$ is determined by the loading strategy $\mathbf{u}$, such that

$$
p_{t} \equiv p\left(r_{t}^{N}\right)
$$

with $r_{t}^{N} \triangleq \frac{1}{C^{N}}\left(D_{t}+\sum_{n \in \mathcal{N}} u_{n t}^{E V}+\sum_{m \in \mathcal{M}} u_{m t}^{N, B L}\right)$ where $C^{N}$ denotes the generation capacity of the grid.

Due to the computational complexity of optimal coordination strategy for large-population systems, in the remaining of the paper we develop a game based decentralized coordination methodology for the underlying coordination systems.

\section{A. Decentralized coordination controls for infinite EV pop- ulations}

As mentioned earlier, centralized coordination strategies for large-population systems require significant networking and centralized computing resources, and may have difficulty gaining public acceptance. Therefore, in the rest of the paper we will study an alternative decentralized game-based charging strategy for large EV populations. This subsection develops the mathematical framework for this analysis, and establishes the conditions required for a Nash equilibrium.

Consider the local cost function $J_{k}$ for an individual agent $k$, with $k \in \mathcal{N} \cup \mathcal{M}$, subject to a collection of strategies $\mathbf{u}$,

$$
J_{k}(\mathbf{u}) \triangleq \sum_{t \in \mathcal{T}} p_{t} u_{k t} .
$$

where the price $p$ is specified in (3).

The locally optimal charging control problem with respect to a fixed collection of controls $\mathbf{u}_{-k}$ is given by the minimization,

$$
V_{k}\left(\mathbf{u}_{-k}\right) \triangleq \inf _{\mathbf{u}_{k}} J_{k}\left(\mathbf{u}_{k} ; \mathbf{u}_{-k}\right),
$$

where $\mathbf{u}_{-k} \triangleq\left\{\mathbf{u}_{l} ; l \in \mathcal{N} \cup \mathcal{M}, l \neq k\right\}$. In other words $\mathbf{u}_{-k}$ denotes the collection of control strategies of all agents except the strategy of the agent $k$. If a minimizing function exists, it will be referred to as an optimal control law for the local charging control problem.

A collection of strategies $\left\{\mathbf{u}_{k}^{*} ; k \in \mathcal{N} \cup \mathcal{M}\right\}$ is a Nash equilibrium if each agent $k$ cannot benefit by unilaterally deviating from its individual strategy $\mathbf{u}_{k}^{*}$, i.e.,

$$
J_{k}\left(\mathbf{u}_{k}^{*} ; \mathbf{u}_{-k}^{*}\right) \leq J_{k}\left(\mathbf{u}_{k} ; \mathbf{u}_{-k}^{*}\right),
$$

for all $\mathbf{u}_{k}$ and all $k \in \mathcal{N} \cup \mathcal{M}$.

In this paper we will study the properties of systems where (i) the number of EV agents is sufficiently large such that the behavior of an individual EV agent on the system is negligible but the behavior of the aggregation of agents can be significant; (ii) the number of bulk load agents (or called 
major agent) is small but the behavior of each of the bulk loads is significant on the grid. To obtain theoretical results, we will examine the asymptotic properties of game systems in the EV population limit.

In particular we consider the following asymptotic assumptions as the population size of EVs $N$ goes to infinity:

$$
\frac{C^{N}}{N} \underset{N \rightarrow \infty}{\longrightarrow} c, \quad \frac{D_{t}^{N}}{N} \underset{N \rightarrow \infty}{\longrightarrow} d_{t}, \quad \frac{\Gamma_{m}^{N, B L}}{N} \underset{N \rightarrow \infty}{\longrightarrow} \Gamma_{m}^{B L} .
$$

Under the asymptotic assumption (7), we observe that any admissible loading strategy $\mathbf{u}_{m}^{N, B L}$ of major agent $m$, specified in (2), satisfies the following:

$$
\frac{1}{N} \sum_{t \in \mathcal{T}} u_{m t}^{N, B L} \underset{N \rightarrow \infty}{\longrightarrow} \Gamma_{m}^{B L}
$$

then we may define a normalized (admissible) loading strategy for major agent $m$, denoted $\mathbf{u}_{m}^{N, B L}$, for the population limit problems, such that

$$
u_{m t}^{B L} \triangleq \lim _{N \rightarrow \infty} \frac{u_{m t}^{N, B L}}{N} .
$$

It is direct to verify that $\mathbf{u}_{m}^{B L}$, in the population limit systems, satisfies the following:

$$
u_{m t}^{B L} \geq 0, \quad \text { and } \quad \sum_{t \in \mathcal{T}} u_{m t}^{B L}=\Gamma_{m}^{B L} .
$$

which is a normalized version of admissible loading strategy given in (2).

Moreover following the above population limit asymptotic assumptions (7), we obtain that, in the infinite population limit of EVs,

$$
\lim _{N \rightarrow \infty} r_{t}^{N}=\frac{1}{c}\left(d_{t}+\sum_{m \in \mathcal{M}} u_{m t}^{B L}+\overline{\mathbf{u}}_{t}^{E V}\right)
$$

where $\overline{\mathbf{u}}_{t}^{E V} \triangleq \frac{1}{N} \sum_{n \in \mathcal{N}} u_{n t}^{E V}$, i.e. $\overline{\mathbf{u}}_{t}^{E V}$ denotes the average charging rate of individual EVs.

\section{B. Distributed mechanism to compute NE with large- population agents}

In this paper we propose a mixed best strategy update mechanism to compute the NE. Essentially all of the individual EV agents simultaneously update their best individual best response (or greedy response) with respect to the strategies of all major agents and an average, or normalized aggregated, charging strategy of the whole EV population. This procedure continues until they do not update their best strategies any more. After the iterative procedure of EV populations, each of major agents sequentially updates his/her best strategy respectively with respect to the proposed aggregated strategy of EV populations, and loading behaviors of the rest of the major agent populations.

Since all of the EV agents simultaneously update their best strategy, the cheap and expensive resources during the charging interval may become expensive and cheap ones respectively. This resulting oscillating behavior implies that the system does not converge to any equilibrium. To mitigate this oscillation behavior of the EV agents we add to the EV agent cost function a quadratic term for the deviation of the individual control strategy from the population average. In this case in the rest of the paper we consider the following revised local cost function:

$$
J_{n}(\mathbf{u}) \triangleq \sum_{t \in \mathcal{T}}\left(p\left(r_{t}\right) u_{n t}^{E V}+\delta\left(u_{n t}^{E V}-\overline{\mathbf{u}}_{t}^{E V}\right)^{2}\right),
$$

for all $n \in \mathcal{N}$, with $r_{t}=\frac{1}{c}\left(d_{t}+\overline{\mathbf{u}}_{t}^{E V}+\sum_{m \in \mathcal{M}} \mathbf{u}_{m t}^{B L}\right)$, where $\delta$ determines the magnitude of the penalty for deviating from the mass average of the whole populations of EVs. It will be shown that the presence of the squared deviation term ensures convergence to a unique collection of locally optimal charging strategies. Furthermore, we will see that the cost added due to this term can be quite small compared with the electricity price $p$.

In this paper we will study the properties of systems where the number of EVs is sufficiently large that the action of an individual $\mathrm{EV}$ on the system is negligible but the action of the aggregation of EVs can be significant. Given the definition of a Nash equilibrium, and the tracking cost function (9) for each $\mathrm{EV}$, we can now establish the conditions governing a Nash equilibrium for an infinite population of EVs.

Theorem 2.1: A collection of charging strategies $\mathbf{u} \equiv$ $\left(\mathbf{u}^{E V} ; \mathbf{u}^{B L}\right)$ for an infinite population of EVs is a Nash equilibrium, if

(i) For all $n \in \mathcal{N}, \mathbf{u}_{n}^{E V}$ minimizes the following cost function,

$$
J_{n}\left(\mathbf{u}_{n}^{E V} ; \mathbf{z}, \mathbf{u}^{B L}\right)=\sum_{t \in \mathcal{T}}\left(p\left(r_{t}\right) u_{n t}^{E V}+\delta\left(u_{n t}^{E V}-z_{t}\right)^{2}\right)
$$

with $r_{t}=\frac{1}{c}\left(d_{t}+z_{t}+\sum_{m \in \mathcal{M}} \mathbf{u}_{m t}^{B L}\right)$, with respect to a fixed $\mathbf{z}$, and $z_{t}=\overline{\mathbf{u}}_{t}^{E V}$, for all $t \in \mathcal{T}$, i.e., $\mathbf{z}$ can be reproduced by averaging the individual optimal charging trajectories of all EVs.

(ii) For all $m \in \mathcal{M}, \mathbf{u}_{m}^{B L}$ minimizes the cost function,

$$
J_{m}\left(\mathbf{u}_{m}^{B L} ; \mathbf{z}, \mathbf{u}_{-m}^{B L}\right)=\sum_{t \in \mathcal{T}} p\left(r_{t}\right) u_{m t}^{B L} .
$$

Note: Theorem 2.1 can be shown following the same technique applied in Theorem 3.1, [5].

Interpretation of Theorem 2.1: A collection of coordination strategies, for game system with limit EV population limit, is a Nash equilibrium, if (i) each of the EVs reacts optimally with respect to a commonly observed charging trajectory that is the aggregation of all EV strategies and the strategies of all major agents; and (ii) each of the major agents reacts optimally with respect to the EV aggregation strategy and the strategies of the rest of major agents. This aggregation strategy is the solution to a fixed point problem in the limit of infinite EV populations.

The loading coordination games studied in the paper are consistent with the mean-field games with major agents studied in [16]. The key similarity is that individual minor EV agents do not consider the behavior of other EV individuals and EV individuals are influenced by the so-called 'mass effect' of the whole population of EVs and major agents, 
while the strategy of each of the major agents is significant. In particular in the case of loading coordination problems, the effect felt by all EV individuals and major load agents is the electricity price, which we specify as a function of the mass average charging trajectory $\overline{\mathbf{u}}_{t}^{E V}$ of whole populations of EVs and loading behavior $\mathbf{u}^{B L}$ of major agents.

\section{Properties of equilibrium of infinite-population systems}

We denote $\widehat{\mathcal{T}}(\mathbf{u})$ as a subset of $\mathcal{T}$ subject to a strategy $\mathbf{u}$, such that

$$
\widehat{\mathcal{T}}(\mathbf{u}) \triangleq\left\{t \in \mathcal{T}, \text { s.t. } \bar{u}_{t}^{E V}>0\right\} .
$$

Also for notational simplicity, we consider $\Sigma(\mathbf{u})$ denotes the total demand subject to strategy $\mathbf{u}$, i.e.

$$
\Sigma\left(\mathbf{u}_{t}\right) \equiv \bar{u}_{t}^{E V}+\sum_{m \in \mathcal{M}} u_{m t}^{B L}, \quad \text { for all } t \in \mathcal{T} .
$$

Theorem 2.2: Consider that the electricity price $p(r)$ is convex and strictly increasing on $r$; then the Nash equilibrium $\mathbf{u}^{*}$ satisfies the following nearly valley-fill property:

$$
\begin{aligned}
& d_{t}+\Sigma\left(\mathbf{u}_{t}^{*}\right)=D, \quad u_{m t}^{*, B L}=a_{m}, \text { for all } t \in \widehat{\mathcal{T}} \\
& d_{t}+\Sigma\left(\mathbf{u}_{t}^{*}\right) \leq d_{s}+\Sigma\left(\mathbf{u}_{s}^{*}\right), \quad \bar{u}_{t}^{E V} \geq \bar{u}_{s}^{E V}, \quad u_{m t}^{B L} \geq u_{m s}^{B L},
\end{aligned}
$$

in case $d_{t} \leq d_{s}$, for all $m \in \mathcal{M}$.

The proof of Theorem 2.2 is given in [17] and follows the similar technique applied in the proof of Theorem 6.1 in [5] where the valley-fill property of Nash equilibrium of the charging games with large populations of minor EV agents is proved.

Note: The 2nd and 3rd part of (12) imply that, at any equilibrium $\mathbf{u}^{*}, \Sigma\left(\mathbf{u}_{t}^{*}\right) \geq \Sigma\left(\mathbf{u}_{s}^{*}\right)$ in case $d_{t} \leq d_{s}$.

\section{Computation mechanism of equilibrium of infinite- population systems}

We now specify an algorithm as below to iteratively compute the $\mathrm{NE}$ for the resource allocation games with infinite population limit, such that all of the individual EV (minor) agents simultaneously update their own best strategy with respect to the mass behavior of whole EV population and demanding strategies of major major agents; and each of major agents update his own best strategy with respect to the mass behavior of EV population and individual behaviors of the rest of major agents successively. We formalize the algorithm as follows:

Algorithm 2.1: We design a mixed iterative computation mechanism for infinite-population games with individual tracking cost function (9) for $\mathrm{EV}$ populations and cost function (5) for major agents:

(S0) Consider an initial collection of average mass demanding strategies of infinite EV populations $\overline{\mathbf{u}}^{E V}$ and loading strategies of major agents $\mathbf{u}^{B L} \equiv\left(\mathbf{u}_{1}^{B L}, \cdots, \mathbf{u}_{M}^{B L}\right)$;

(S1) Update the best strategies of individual EV agents as follows:

(S1.1) All of the individual EV agents simultaneously implement their own best strategies $\left(\mathbf{u}_{n}^{E V, *} ; n \in \mathcal{N}\right)$ each of which respectively minimizes the tracking cost function (13) specified below, with respect to $\left(\overline{\mathbf{u}}^{E V} ; \mathbf{u}^{B L}\right)$,

$$
\begin{aligned}
& J_{n}\left(\mathbf{u}_{n}^{E V} ; \overline{\mathbf{u}}^{E V} ; \mathbf{u}^{B L}\right) \\
= & \sum_{t \in \mathcal{T}}\left(p\left(r_{t}\right) u_{n t}^{E V}+\delta\left(u_{n t}^{E V}-\overline{\mathbf{u}}^{E V}\right)^{2}\right) ;
\end{aligned}
$$

(S1.2) Update $\overline{\mathbf{u}}^{E V}$ with the average value of collection of best strategies $\left(\mathbf{u}_{n}^{E V, *} ; n \in \mathcal{N}\right)$ implemented in $(\mathrm{S} 1.1)$;

(S1.3) Repeat (S1.1) and (S1.2) until $\overline{\mathbf{u}}^{E V}$ can not be updated any more.

(S2) Update the best strategies of each of the major agents successively as below:

(S2.1) Implement the best strategy $\mathbf{u}_{m}^{B L, *}$ of major agent $m$, with $m \in \mathcal{M}$, which minimizes the cost function

$$
J_{m}\left(\mathbf{u}_{m}^{B L} ; \overline{\mathbf{u}}^{E V} ; \mathbf{u}_{-m}^{B L}\right)=\sum_{t \in \mathcal{T}} p\left(r_{t}\right) u_{m t}^{B L},
$$

where $\mathbf{u}_{-m}^{B L} \equiv\left(\mathbf{u}_{k}^{B L} ; k \neq m, k \in \mathcal{M}\right)$;

(S2.2) Update $\mathbf{u}_{m}^{B L}$ in $\left(\mathbf{u}_{1}^{B L}, \cdots, \mathbf{u}_{m}^{B L}, \cdots, \mathbf{u}_{M}^{B L}\right)$ with $\mathbf{u}_{m}^{B L, *}$ specified in (S2.1);

(S2.3) Implement (S2.1) and (S2.2) successively for all $m \in$ $\mathcal{M}$.

(S3) Repeat (S1) and (S2) until $\left(\overline{\mathbf{u}}^{E V} ; \mathbf{u}^{B L}\right)$ can not be updated.

Notes: (i) By applying Theorem 4.2 in [5] a sufficient condition under which the update algorithm (S1) converges is that the tracking parameter $\delta$ in (9) satisfies the following inequality constraint:

$$
\frac{1}{2 c} \sup _{r \in\left[r_{\min }, r_{\max }\right]} \frac{d p}{d r} \leq \delta \leq \frac{a}{c} \inf _{r \in\left[r_{\min }, r_{\max }\right]} \frac{d p}{d r} .
$$

(ii) In case that the update procedure specified in $\mathrm{Al}$ gorithm 2.1 converges, the proposed collection of agent best strategies $\left(\mathbf{u}^{E V, *} ; \mathbf{u}^{B L, *}\right)$ is an $\mathrm{NE}$ for the underlying infinite-population game systems. As demonstrated in the next section, considering some proper specifications, the game system converges to the Nash equilibrium following Algorithm 2.1.

\section{NUMERICAL EXAMPLES}

A few of examples will be used in this section to illustrate the main results developed in Section II. The examples use the base demand profile of Figure 1, which shows a normalized base demand in a typical summer day, from noon on one day to noon on the next. It is assumed that the normalized total generation capacity $c$ is $12 \mathrm{~kW}$.

We consider identical battery size $\Gamma_{n}^{E V}=10 \mathrm{kWh}$ for all EVs, and suppose that there exist two bulk load units and each of the normalized energy demands for these bulk loads $\Gamma_{1}^{B L}$ and $\Gamma_{2}^{B L}$ is equal to $2 \mathrm{kWh}$ and $3 \mathrm{kWh}$ respectively.

The common charging interval for all EVs and bulk load units, $\mathcal{T}$, covers the 12 -hour period from $8: 00 \mathrm{pm}$ on one day to $8: 00 \mathrm{am}$ on the next. The continuously differentiable and strictly increasing price function

$$
p(r)=0.15 r^{1.1} \$ / \mathrm{kWh}
$$




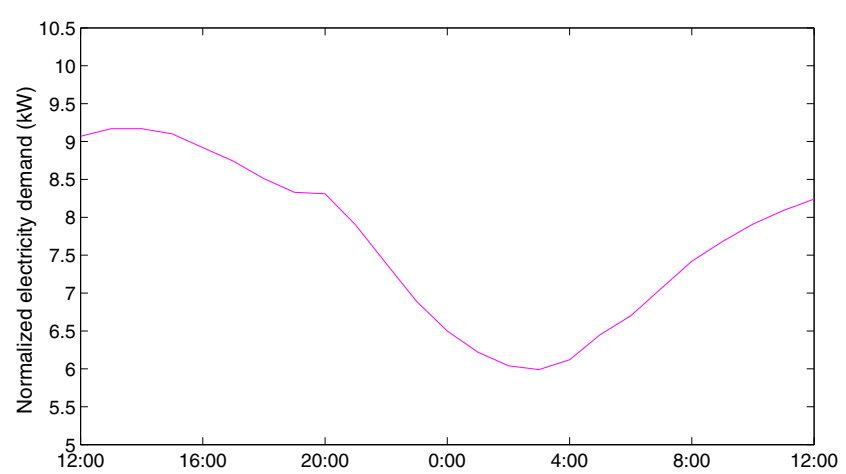

Fig. 1. A normalized base demand in a typical summer day.

is used in all cases.

We also consider that the tracking cost parameter $\delta$ equals 0.01 . As analyzed in the below, the $\delta$ satisfies the inequality constraint (14).

It may be verified from Figure 1 that

$$
r_{\min }=\min _{t \in \mathcal{T}}\left\{d_{t}\right\} / c \approx 0.5 .
$$

To determine $r_{\max }$, we assume the entire energy requirement from EVs and bulk load units is delivered over a single time step, so

$$
r_{\max }=\frac{1}{c}\left(\max _{t \in \mathcal{T}}\left\{d_{t}\right\}+\Gamma^{E V}+\Gamma_{1}^{B L}+\Gamma_{2}^{B L}\right) \approx 2 .
$$

where $\Gamma^{E V}$ represents the common value of $\Gamma_{n}^{E V}$ for all $n \in \mathcal{N}$ and is equal to $10 \mathrm{kWh}$.

Referring to (15), this gives

$$
\frac{1}{2 c} \max \frac{d p(r)}{d r}=0.0074 \leq \frac{a}{c} \min \frac{d p(r)}{d r}=0.0128 a,
$$

with some $a$ in the range $\frac{1}{2}<a<1$, which verifies the inequality constraint (14) with the tracking parameter $\delta$ of 0.01 .

Figures $2-4$ below provide simulation results for the decentralized computation algorithm (S1)-(S3) specified in last section.

First, Figure 2 displays the best strategy update procedure for EV populations following mechanism (S1.1)-(S1.3) with respect to the non-EV demand composed of the base demand and zero initial demands of major agents. It can be observed that, in a few of update iterations, the EV populations converge to the valley-fill charging strategy, the line marked with dots in Figure 2.

Next, by applying the mixed update mechanism, the first major agent need to update his best strategy with respect to the updated EV charging strategy, given in Figure 2, and the given initial demanding from 2nd major agent. Figure 3 demonstrates this best demanding update of first major agent. which is a nearly valley fill strategy and fits with Theorem 2.2.

Following the major agent 1 , the major agent 2 needs to updates his strategy. Figure 4 displays the best demanding update from major agent 2 with respect to the updated

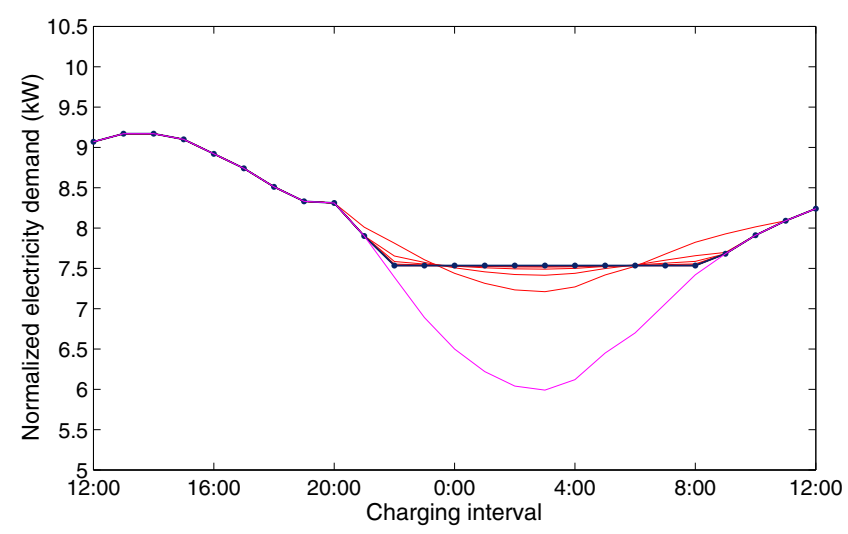

Fig. 2. Best strategy update iterations of EV populations by applying Algorithm (S1.1)-(S1.3) with respect to zero initial demands of all agents.

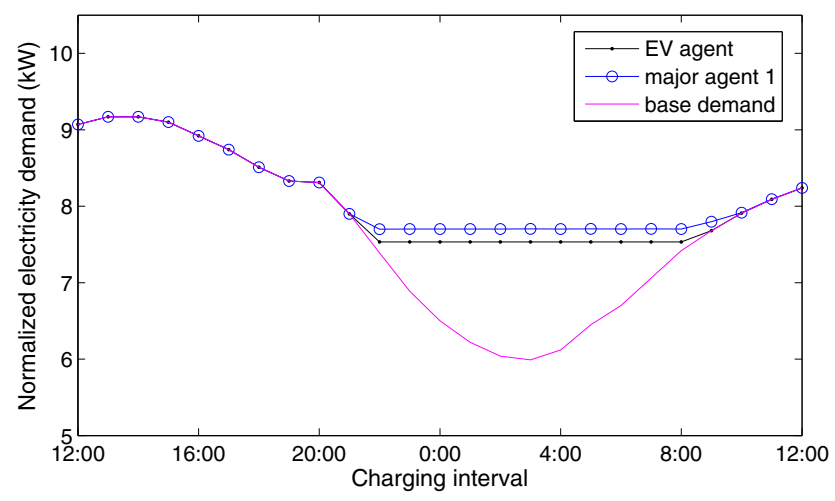

Fig. 3. Best strategy updates of 1st major agent with respect to initial demands of 2nd major agent and mass charging strategies of EV populations illustrated in Figure 2.

demanding strategy of 1st major agent, the updated mass charging strategy of EV populations illustrated in Figure 3 and Figure 2 respectively.

For this specific example, the system reaches the Nash equilibrium as displayed in Figure 4 after a single update step.

Besides the example with initial agent demand given above where the system converges to a Nash equilibrium in single iterative step, next we demonstrate the implementation of Nash equilibrium with non-zero initial agent demand illustrated in Figure 5.

Figure 6 displays the aggregate demand of the best strategy updates of all agents in each of the update iterations with the initial demand displayed in Figure 5. It can be observed that the system converges to the same nearly valley-fill strategy as that with zero initial agent demands, while the iterative steps may vary with respect to the initial demand of agents.

\section{Conclusions And FUture Research}

This paper specifies a class of loading coordination problems for large populations of EVs and a few of bulk load units. These problems are formulated as large-population dynamic games on a finite interval. We studied the valleyfill property of the Nash equilibrium for the underlying 


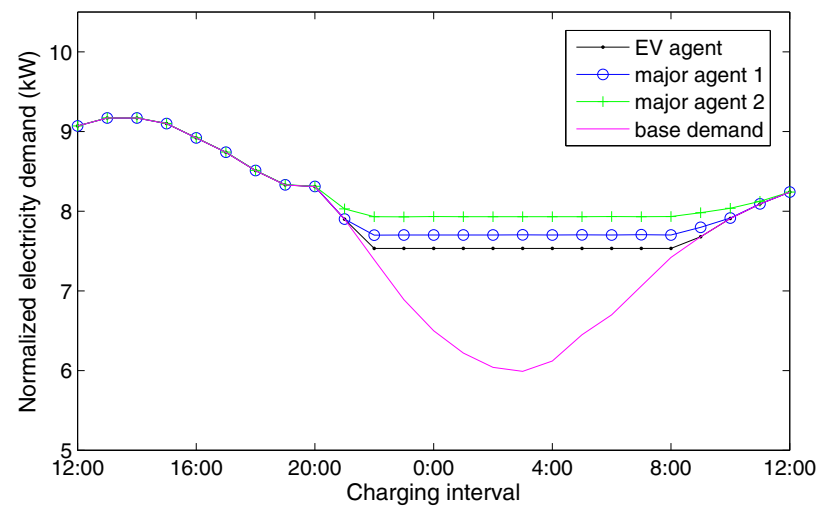

Fig. 4. Best strategy updates of 2nd major agent with respect to the updated loading strategies of 1st major agent given in Figure 3 and mass charging strategies of EV populations illustrated in Figure 2.

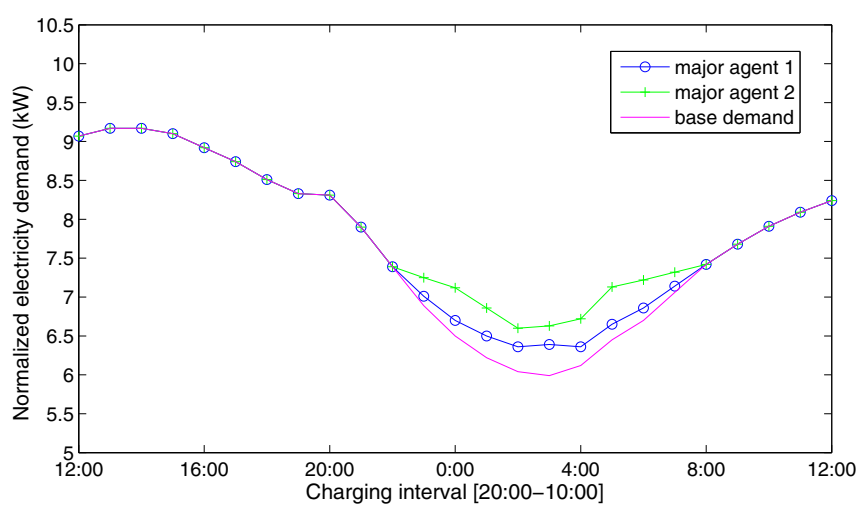

Fig. 5. A specific non-zero initial major agent demand.

problems. In particular, as demonstrated in numerical examples, following a computational algorithm and under certain conditions, the system converges to a nearly valley-fill Nash equilibrium.

For the sake of analytical simplicity, at the current stage, we consider that all of the EVs share the same specifications, each loading unit will be fully charged at the terminal instant, and base demand as well as electricity supply are deterministic and predictable. Future work should release these assumptions to facilitate the method applicable in real large population loading coordination problems.

\section{REFERENCES}

[1] P. Denholm and W. Short, "An evaluation of utility system impacts and benefits of optimally dispatched plug-in hybrid electric vehicles," National Renewable Energy Labortary, Technical Report NREL/TP620-40293, October 2006.

[2] S. Rahman and G. Shrestha, "An investigation into the impact of electric vehicle load on the electric utility distribution system," IEEE Transactions on Power Delivery, vol. 8, no. 2, pp. 591-597, 1993.

[3] F. Koyanagi and Y. Uriu, "Modeling power consumption by electric vehicles and its impact on power demand," Electrical Engineering in Japan, vol. 120, no. 4, pp. 40-47, 1997.

[4] F. Koyanagi, T. Inuzuka, Y. Uriu, and R. Yokoyama, "Monte Carlo simulation on the demand impact by quick chargers for electric vehicles," in IEEE Power Engineering Society Summer Meeting, vol. 2, 1999.

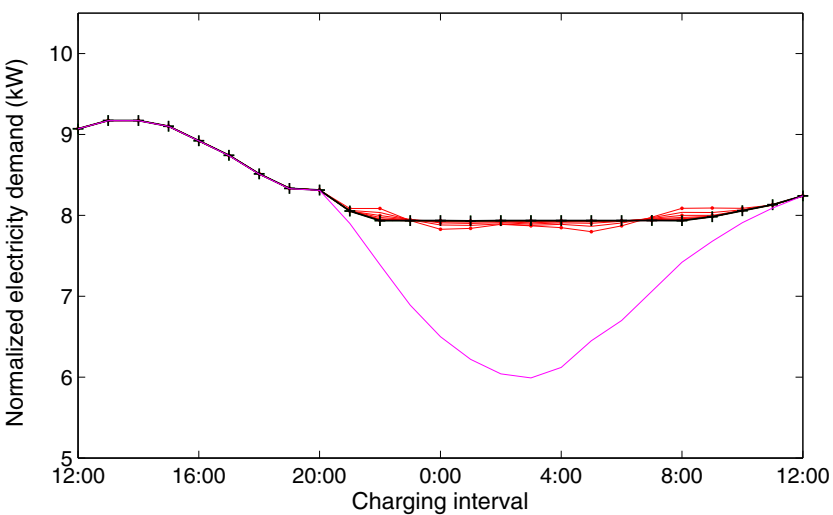

Fig. 6. Total demand updates of all agents for game systems with the non-zero initial major agent demands displayed in Figure 5.

[5] Z. Ma, D. Callaway, and I. Hiskens, "Decentralized charging control of large populations of plug-in electric vehicles," Accepted by IEEE Trans. on Contr. Syst. Tech., 2011.

[6] Z.Ma, D.Callaway, and I.Hiskens, "Decentralized charging control for large populations of plug-in electric vehicles: Application of the nash certainty equivalence principle," in In the proceedings of the 2010 IEEE Multi-Conference on Systems and Control, Yokohama, Japan, September 2010, pp. 191-195.

[7] _ , "Decentralized charging control for large populations of plug-in electric vehicles," in In the proceedings of the 49th IEEE Int. Conf. Decision and Control, Atlanta, GA, US, December 2010.

[8] R.A.Waraich, M.Galus, C.Dobler, M.Balmer, G.Andersson, and K.Axhausen, "Plug-in hybrid electric vehicles and smart grid: Investigations based on a micro-simulation," Institute for Transport Planning and Systems, ETH Zurich, Switzerland, Technical Report 10.3929/ethza-005916811, 2009.

[9] G. Christodoulou, V. Mirrokni, and A. Sidiropoulos, "Convergence and approximation in potential games," in in Proceedings of the 23rd Symposium on Theoretical Aspects of Computer Science (STACS), 2006, pp. 349-360.

[10] E. Even-Dar, A. Kesselman, and Y. Mansour, "Convergence time to nash equilibirum in load balancing," ACM Transactions on Computational Logic, vol. 2, pp. 111-132, 2001.

[11] P.Berenbrink, T.Friedetzky, L.Goldberg, P.Goldberg, Z.Hu, and R.Martin, "Distributed selfish load balancing," in in Proceedings of the 17th Annual ACM-SIAM Symposium on Discrete Algorithms, Miami, Florida, January 22-26 2006, pp. 354-363.

[12] A.Blum, E.Even-Dar, and K.Ligett, "Routing without regret: On convergence to nash equilibria of regret-minimizing algorithms in routing games," in in Proceedings of the 25th ACM Symposium on Principles of Distributed Computing, 2006, pp. 45-52.

[13] S.Fischer, L.Olbrich, and B.Vocking, "Approximating Wardrop equilibria with finitely many agents," Distributed Computing, vol. 21(2), pp. 129-139, 2008.

[14] S.Fischer, H.Racke, and B.Vocking, "Fast convergence to Wardrop equilibria by adaptive sampling methods," in Proceedings of the thirtyeighth annual ACM symposium on Theory of computing, Seattle, WA, May 21-23 2006, pp. 653-662.

[15] J.Wardrop, "Some theoretical aspects of road traffic research," in in Proceedings of the Institution of Civil Engineers, 1952, pp. 325-78.

[16] M. Huang, "Large-population LQG games involving a major player: the Nash certainty equivalence principle," SIAM Journal on Control and Optimization, vol. 48, pp. 3318-3353, 2010.

[17] Z.Ma, Y.Duan, and L.Dong, "Decentralized loading coordinations for large-population heterogeneous plug-in electric vehicles and a few controllable bulk loads," Beijing Institute of Technology," Technical Report, September 2011. 